\title{
Síndrome de bajo gasto cardíaco en el posoperatorio de cirugía cardíaca
}

\author{
Dr. Sebastián Lorenzo
}

\section{Resumen}

El síndrome de bajo gasto cardíaco, como su nombre lo indica, comprende un conjunto de signos y síntomas determinados por la incapacidad del sistema cardiovascular de satisfacer la demanda metabólica tisular debido a un bajo volumen minuto. Es una complicación frecuente en el posoperatorio de cirugía cardíaca, y abarca un gran espectro en su presentación, desde un cuadro de corta duración, controlable con drogas vasoactivas e inotrópicas, hasta el shock cardiogénico refractario de gran mortalidad. Aumenta los tiempos de internación, la morbimortalidad a corto y largo plazo, y la utilización de recursos sanitarios. Pese al avance tecnológico en la cardiología intervencionista y terapia intensiva, no deja de ser una complicación frecuente y en ocasiones de muy difícil manejo, siendo fundamental comprender su fisiopatología, etiología y presentación clínica para un adecuado manejo del paciente durante su etapa de recuperación cardiovascular. En esta revisión repasaremos estos temas, y mencionaremos conceptos clave para el tratamiento de este síndrome.

Palabras clave:

POSOPERATORIO CIRUGÍA CARDÍACA

BAJO GASTO CARDÍACO

MONITOREO HEMODINÁMICO

\section{Low cardiac output syndrome in the postoperative period of cardiac surgery}

\section{Summary}

Low cardiac output syndrome comprises a set of signs and symptoms determined by the inability of the cardiovascular system to satisfy tissue metabolic demand due to low minute volume. It is a frequent complication in the postoperative period of cardiac surgery, and its presentation covers a wide spectrum, from a short-term condition controllable with vasoactive and inotropic drugs to refractory cardiogenic shock with high mortality. It increases hospitalization times, short and long-term morbidity and mortality, and health resources usage. Despite the technological advance in interventional cardiology and intensive therapy, it is still a frequent complication and sometimes very difficult to manage, being essential to understand its pathophysiology, etiology and clinical presentation for an adequate management of the patient during his cardiovascular recovery stage. In this review we will analyze some key concepts for the treatment of this syndrome.

Key words:

\author{
POSTOPERATIVE PERIOD CARDIAC SURGERY \\ LOW CARDIAC OUTPUT \\ HEMODYNAMIC MONITORING
}

\section{Síndrome de baixo débito cardíaco no pós-operatório de cirurgia cardíaca}

\section{Resumo}

A síndrome de baixo débito cardíaco, como o próprio nome sugere, compreende um conjunto de sinais e sintomas determinados pela incapacidade do sistema cardiovascular em satisfazer a demanda metabólica dos tecidos devido ao baixo volume minuto. É uma complicação frequente no pós-operatório de cirurgia cardíaca e sua apresentação abrange um amplo espectro, desde uma condição de curta duração, controlável com drogas vasoativas e inotrópicas, até choque cardiogênico refratário com alta mortalidade. Aumenta o tempo de hospitalização, a morbimortalidade de curto e longo prazo e a utilização de recursos de saúde. Apesar do avanço tecnológico na cardiologia intervencionista e na terapia intensiva, ainda é uma complicação frequente e algumas vezes de difícil manejo, sendo fundamental o entendimento de sua fisiopatologia, etiologia e apresentação clínica para um manejo adequado do paciente durante sua recuperação cardiovascular. Nesta revisão mencionaremos os conceitos-chave para o tratamento dessa síndrome.

$\begin{array}{ll}\text { Palavras-chave: } & \text { PÓS-OPERATÓRIO DE CIRURGIA CARDÍACA } \\ & \text { BAIXO DÉBITO CARDÍACO } \\ & \text { MONITORIZAÇAO HEMODINÂMICA }\end{array}$

Centro de Investigación Cardiovascular Uruguayo (CICU), Casa de Galicia. Montevideo, Uruguay.

Correspondencia: Dr. Sebastián Lorenzo. Correo electrónico: lorenzoseba@hotmail.com

El autor declara no tener conflictos de intereses.

Recibido Oct 5, 2020; aceptado Oct 27, 2020 


\section{Introducción}

El manejo posoperatorio en terapia intensiva de los pacientes cardiológicos ha sido un eslabón fundamental para la recuperación cardiovascular luego de cirugía, considerado incluso como una subespecialidad dentro de la cardiología.

Fenómenos como la isquemia-reperfusión, el daño inflamatorio producido por la cardioplejia y la bomba de circulación extracorpórea (CEC), la anemia, la asistencia respiratoria mecánica y la medicación anestésica, entre otros, generan un estrés que se suma a la disfunción miocárdica previa, determinando una nueva situación para el sistema cardiovascular, con la consecuente disminución del gasto cardíaco (GC) que puede comprometer el pronóstico del paciente y aumentar la mortalidad. La falla renal, la prolongación de los tiempos de asistencia respiratoria mecánica y la estancia prolongada en terapia intensiva son algunas de las consecuencias de este síndrome. Su presentación clínica es variada y su etiología generalmente multifactorial; a menudo se acompaña de otras disfunciones que agravan e incluso perpetúan el cuadro. El descenso del volumen minuto en este contexto constituye un desafío para el médico que recibe al paciente en terapia intensiva y es clave el entendimiento de la etiopatogenia y fisiopatología para un manejo adecuado.

\section{Definición}

Si bien el síndrome fue descrito por primera vez en 1975 por Parrs y colaboradores ${ }^{(1)}$, aún no hay consenso sobre su exacta definición, encontrando diversas formas de catalogarlo en la bibliografía. En algunos trabajos se define según la necesidad de balón de contrapulsación intraaórtico (BIAC) o apoyo inotrópico por cierto período de tiempo para mantener determinados parámetros hemodinámicos ${ }^{(2,3)}, \mathrm{y}$ en otros solo por signos clínicos ${ }^{(4)}$. También se define según el índice cardíaco (IC), con diferencias en el punto de corte según la bibliografía, asociado a presión de enclavamiento capilar pulmonar (PCP) elevada. Según el documento de consenso de la Sociedad Española de Medicina Intensiva, Crítica y Unidades Coronarias (SEMICYUC) publicado en $2012^{(5)}$, se define como un IC $<2,2 \mathrm{l} / \mathrm{min} / \mathrm{m}^{2}$, sin hipovolemia relativa asociada (entendida como PCP $<15 \mathrm{mmHg}$ ). Dado que en la práctica clínica diaria no siempre conocemos el GC, se define también el "cuadro clínico compatible con síndrome de bajo gasto cardíaco" (SBGC), caracterizado por oliguria, saturación venosa central $<60 \%$ (y saturación arterial normal) y/o lactato $>3 \mathrm{mmol} / \mathrm{l}$, sin hipovolemia relativa, incluyéndose también los pacientes que re- quieren inotrópicos y/o BIAC para mantener un adecuado estado hemodinámico. También define el shock cardiogénico cuando el IC es $<2,0 \mathrm{l} / \mathrm{min} / \mathrm{m}^{2}$, sin hipovolemia relativa, con oliguria y presión arterial sistólica $<90 \mathrm{mmHg}$.

Si bien esta última definición es útil, ya que acepta el término de SBGC en pacientes en los que no conocemos el GC, (muy frecuente en la práctica clínica habitual) incluye el concepto de "hipovolemia relativa". Según la literatura, esta se define como la incapacidad del volumen sanguíneo de llenar el compartimiento vascular por disminución de las resistencias vasculares sistémicas (RVS) ${ }^{(7,8)}$. Sin embargo, la definición de la SEMICYUC se refiere a una PCP $<15 \mathrm{mmHg}$.

Este concepto de "hipovolemia relativa", al incluirse en la definición de SBGC, más que simplificar el encare clínico, excluye algunas situaciones que cursan con GC disminuido:

- Por un lado, como veremos más adelante, el SBGC muchas veces es consecuencia de un fallo del ventrículo derecho (VD) ${ }^{(6)}$, el cual se asocia a PCP normales o bajas sin cursar con hipovolemia relativa.

- A su vez, en ocasiones, los pacientes con SBGC también presentan un verdadero estado de hipovolemia relativa, determinado por vasoplejia, a menudo vinculado a la bomba de CEC (aunque se ha visto en pacientes sin $\mathrm{CEC})^{(8)}$.

Por lo tanto, la hipovolemia relativa no excluye la presencia de SBGC, y muchas veces coexiste con causas que sí disminuyen el GC.

\section{Incidencia y morbimortalidad}

En relación a la incidencia, está en gran parte determinada por el criterio diagnóstico utilizado, y en la literatura encontramos desde $9,1 \%^{(2)}$ hasta $25 \%$ (1).

La morbimortalidad también se ha visto aumentada en pacientes que cursan con bajo GC. En el estudio de Rao y colaboradores, la mortalidad fue de $17 \%$ en el subgrupo con SBGC comparado con 0,9\% en el subgrupo sin $\mathrm{SBGC}^{(2)}$. En el estudio publicado por WenJun Ding y colaboradores ${ }^{(9)}$, la presencia de SBGC se asoció a mayor estancia en CTI e internación total, mayor tasa de falla respiratoria, ataque cerebrovascular, falla renal aguda, y mortalidad a 30 días. En otro trabajo también fue mayor la mortalidad en el subgrupo de pacientes con $\mathrm{SBGC}^{(3)}$. A su vez, se ha visto que la mortalidad por SBGC ha aumentado en los últimos 30 años, lo que podría explicarse porque actualmente se intervienen pacientes de mayor riesgo operatorio ${ }^{(10)}$. 


\section{Factores predisponentes}

La probabilidad de que un paciente desarrolle el síndrome depende de numerosos factores tanto preoperatorios como propios de la cirugía, los cuales predisponen a la falla cardíaca.

En un trabajo unicéntrico publicado en 2015 que incluyó 1.524 pacientes sometidos a revascularización miocárdica aislada, de los cuales 205 desarrollaron SBGC según criterios clínicos, se concluyó mediante análisis multivariado que la edad $>65$ años (OR = 1,85; IC 95\% 1,27-3,76; $\mathrm{p}=0,001)$, fracción de eyección del ventrículo izquierdo (FEVI) $<50 \%$ (OR $=2,05$; IC 95\% 1,53-4,54; $\mathrm{p}=0,005)$, cirugía con CEC (OR = 2,16; IC 95\% 1,53-4,86; $\mathrm{p}=$ 0,018 ), la conversión de cirugía sin bomba a con bomba $(\mathrm{OR}=9,15 ;$ IC $95 \% 3,84-16,49 ; \mathrm{p}<0,0001)$ y la revascularización incompleta (definida según criterios anatómicos como la ausencia de revascularización de todos los vasos $\geq 1 \mathrm{~mm}$ de diámetro) (OR $=2,62 ; \mathrm{IC} 95 \% 1,79-5,15 ; \mathrm{p}=0,007)$ fueron factores independientes de $\mathrm{SBGC}^{(9)}$.

En otro estudio reciente que incluyó 156 pacientes en los que se realizó cirugía de sustitución valvular aórtica, mitral o ambas, se determinó que la disfunción renal preoperatoria $(\mathrm{OR}=5,13 ; \mathrm{p}=0,005)$, el tiempo prolongado de CEC $>120$ minutos (OR= $4,89 ; \mathrm{p}=0,001)$ y la resistencia vascular pulmonar elevada ( $>3 \mathrm{U}$. Wood) $(\mathrm{OR}=7,52 ; \mathrm{p}<0,001)$ fueron las variables que se asociaron de manera independiente a la aparición de bajo gasto(3).

A su vez, la malnutrición se asocia a aumento de la necesidad de inotrópicos en el posoperatorio ${ }^{(6,11)}$ y en pacientes obesos con insuficiencia renal revascularizados con cirugía, la tasa de SBGC fue mayor que en aquellos con insuficiencia renal con índice de masa corporal $<30^{(12)}$.

También el péptido natriurético tipo $\mathrm{B}$ (BNP) preoperatorio y la anemia se asocian a SBGC. En un estudio publicado en 2013, que incluyó pacientes con cirugía cardíaca electiva, el BNP> $258 \mathrm{pg} / \mathrm{ml}$ (OR 3,46; IC 95\% 1,53-7,80; $\mathrm{p}=0,003$ ) y la anemia con hemoglobina $(\mathrm{Hb})$ entre 8,8 y $13,1 \mathrm{~g} / \mathrm{dl}(\mathrm{OR}$ 0,33 ; IC $95 \% 0,13-0,81 ; \mathrm{p}=0,016$ ) se asociaron independientemente a un aumento de la necesidad de inotrópicos en el posoperatorio durante las primeras $24 \mathrm{~h}$, comparado con valores de BNP entre $0-25,2 \mathrm{pg} / \mathrm{ml}$ y de $\mathrm{Hb}$ entre $16,1-20,2 \mathrm{~g} / \mathrm{dl}^{(13)}$. Marcadores inflamatorios como leucocitosis y proteína $\mathrm{C}$ reactiva no se correlacionaron en forma independiente con SBGC.

En otro estudio, el recuento linfocitario preoperatorio $\leq 2.007 \mathrm{cel} / \mu \mathrm{L}$ se asoció de manera independiente al uso de inotrópicos o vasopresores por más de 6 h $(\mathrm{p}<0,001)^{(14)}$.
Otros factores anteriormente conocidos como predisponentes del síndrome ${ }^{(2)}$, tales como hipertensión arterial (HTA), sexo femenino, enfermedad de tres vasos o de tronco coronario izquierdo, actualmente no se consideran como tales ${ }^{(5,6,10)}$.

\section{Fisiopatología}

No es sencillo conceptualizar los mecanismos fisiopatológicos que determinan el síndrome, por su carácter multifactorial, donde entran en juego diversos factores, algunos vinculados a la propia cirugía, y otros preexistentes, que generan daño directamente sobre las células miocárdicas (provocando disfunción miocárdica), y sobre otros sistemas que afectan el GC.

Por un lado existen condiciones preoperatorias los factores predisponentes mencionados previamente -que favorecen el desarrollo del SBGC. La disfunción diastólica previa a la cirugía también predispone al SBGC en el posoperatorio $(\mathrm{PO})^{(8)}$. A su vez, aunque no sean considerados marcadores independientes de SBGC, condiciones como la acidosis, el infarto de miocardio reciente, la isquemia persistente y la elevación de los niveles de troponina ultrasensible, entre otros, se asociaron a mayor probabilidad de desarrollar $\operatorname{SBGC}^{(8,15)}$.

La cirugía con CEC es uno de los principales responsables de una intensa respuesta inflamatoria sistémica, que provoca daño, disfunción celular y mitocondrial, con la consecuente necrosis y apoptosis a nivel miocárdico. Asimismo, los tiempos prolongados de CEC se asocian a mayor uso de inotrópicos durante el $\mathrm{PO}^{(6,16)}$.

A nivel sistémico, la CEC provoca un gran desequilibrio del medio interno, con trastornos hidroelectrolíticos y ácido-base, que afectan directamente la función ventricular. También se alteran la coagulación y la función plaquetaria, determinando un aumento del riesgo de sangrado y sus consecuencias: anemia, plaquetopenia, y eventualmente taponamiento cardíaco ${ }^{(16,17)}$.

Otra complicación que ocurre en las cirugías con paro, está vinculada al fenómeno de isquemia-reperfusión, generalmente provocado por una protección miocárdica deficiente, con la cardioplejia y/o el clampeo-declampeo aórtico. El mismo se desencadena una vez restaurado el flujo sanguíneo normal, tras lo cual se genera una respuesta inflamatoria que induce mayor daño miocárdico ${ }^{(1,18,19)}$. Esto también ocurre a nivel pulmonar, al desvincularse de la CEC, situación a la que nos referimos como injuria pulmonar por isquemia-reperfusión, que se manifiesta por edema pulmonar e hipoxemia ${ }^{(1)}$. 
Tabla 1. Disfunción ventricular.

Disfunción del VD

Bajo gasto

- Elementos clínicos y paraclínicos de hipoperfusión sistémica

- Índice cardíaco $<2,2 \mathrm{l} / \mathrm{min} / \mathrm{m}^{2}$

Presiones de llenado en cavidades izquierdas normales o disminuidas

- $\mathrm{PCP}<18 \mathrm{mmHg}$

Presiones de llenado en cavidades derechas incrementadas

Disfunción del VI

Bajo gasto

- Elementos clínicos y paraclínicos de hipoperfusión sistémica

- Índice cardíaco $<2,2 \mathrm{l} / \mathrm{min} / \mathrm{m}^{2}$

Presiones de llenado en cavidades izquierdas incrementadas

- $\mathrm{PCP}>18 \mathrm{mmHg}$

Presiones de llenado en cavidades derechas normales o disminuidas

- $\mathrm{PVC}<15 \mathrm{mmHg}$

Disfunción biventricular

Bajo gasto

- Datos clínicos y paraclínicos de hipoperfusión sistémica

- Índice cardíaco $<2,2 \mathrm{l} / \mathrm{min} / \mathrm{m}^{2}$

Presiones de llenado en cavidades izquierdas incrementadas

- $\mathrm{PCP}>18 \mathrm{mmHg}$

Presiones de llenado en cavidades derechas incrementadas

- $\mathrm{PVC}>15 \mathrm{mmHg}$

VI: ventrículo izquierdo; VD: ventrículo derecho; PCP: presión capilar pulmonar; PVC; presión venosa central.

La manipulación directa del corazón, la disminución parcial o total de la perfusión coronaria, la revascularización incompleta, complicaciones en las anastomosis o trombosis del injerto, el espasmo coronario y la hipoxemia sistémica, también constituyen causas de isquemia, que pueden llegar a producir infarto transmural con compromiso de la función sistólica ${ }^{(8,16)}$.

Estas alteraciones sumadas a otras, como por ejemplo, la presencia de ventilación con presión po- sitiva, neumotórax a tensión, arritmias, obstrucción del tracto de salida del ventrículo izquierdo (TSVI), reacción a la protamina u otros fármacos anestésicos, hipertensión pulmonar (HTP), tromboembolismo pulmonar (TEP), disfunción valvular persistente o distrés respiratorio, predisponen a la presencia del síndrome ${ }^{(8)}$. Como muchas de estas causas no necesariamente cursan con PCP >15 $\mathrm{mmHg}$, creemos que dicho parámetro no debe incluirse en la definición de SBGC.

Varios de estos factores derivan en la llamada "disfunción miocárdica", uno de los principales mecanismos de SBGC, que se puede presentar inmediatamente tras la desvinculación de la $\mathrm{CEC}^{(20)}$. La misma puede afectar al ventrículo izquierdo (VI), al $\mathrm{VD}$, o a ambos, pudiendo ser sistólica o diastólica ${ }^{(1)}$, transitoria o persistente, y conceptualmente ser consecuencia de alteraciones en la precarga, poscarga o la contractilidad cardíaca ${ }^{(1,8)}$.

Disfunción sistólica del VI: la disminución de la FEVI puede deberse a pérdida de miocitos (por necrosis o apoptosis) o a una disfunción de estos, transitoria o definitiva. Como mencionamos previamente, la disfunción sistólica previa, tanto isquémica como de cualquier otra etiología, predispone a la aparición del síndrome. Los fenómenos provocados durante la cirugía generan un estado de atontamiento, en general transitorio, que puede comprometer el $\mathrm{GC}^{(8)}$. La respuesta a la precarga también está disminuida, y una poscarga aumentada puede causar caída de la función sistólica ${ }^{(6)}$ (tabla 1).

Disfunción diastólica del VI: la alteración de la diástole, tanto en su fase de relajación isovolumétrica como en la de llenado, afecta al GC en el PO. Se ha demostrado que en pacientes sin disfunción diastólica previa, la CEC y la cardioplejia provocan alteración en esta fase del ciclo cardíaco ${ }^{(21)}$, debido al edema miocárdico provocado por el fenómeno de isquemia-reperfusión ${ }^{(8)}$. En otros casos la distensión del VD puede desviar el septum interventricular (SIV) y comprometer el llenado del VI ${ }^{(8)}$. A su vez, el SBGC se presenta en pacientes con disfunción diastólica previa, frecuentemente en pacientes con VI hipertrófico y pequeño; esta es una situación habitual en pacientes de sexo femenino con hipertrofia ventricular izquierda (HVI) por HTA o estenosis aórtica. En este escenario de disfunción diastólica, la presencia de taquiarritmias, asincronía aurículo-ventricular, isquemia o aumento de la poscarga, pueden provocar SBGC ${ }^{(6,8)}$.

Disfunción del VD: se presenta en 0,04\%-1\% de los pacientes ${ }^{(5)}$. Puede deberse a isquemia/infarto, al fenómeno de isquemia-reperfusión y al aumento de la poscarga. El aumento de la poscarga, en los casos de cardiopatías congénitas, puede vincu- 
larse a la presencia de estenosis pulmonar residual $^{(1)}$, aumento de la reactividad vascular pulmonar, embolia pulmonar o aumento de las presiones en cavidades izquierdas ${ }^{(1,6)}$ que ocasiona HTP (frecuente en la cirugía de la válvula mitral)(8). También puede asociarse al compromiso de la contractilidad y posterior dilatación, provocando un aumento en la presión diastólica final del VD, protrusión del SIV al VI, disminución de la precarga del VI, con el consiguiente compromiso del $\mathrm{GC}^{(6)}$.

Por lo tanto, el SBGC podría considerarse como la vía final común de todos estos fenómenos, por lo que su manejo es tan variado como su etiología y presentación clínica (tabla 2).

\section{Presentación clínica}

La clínica está marcada por los elementos de hipoperfusión sistémica(17), y a su vez por signos y síntomas que orientan a la etiología del cuadro. El paciente presenta palidez, livideces, relleno capilar lento, extremidades frías y oligoanuria. En ocasiones puede presentar ingurgitación yugular, congestión pulmonar, hipotensión arterial sistémica y taquicardia sinusal. Se debe controlar el gasto de los drenajes torácicos, que puede estar aumentado de forma constante, o detenerse súbitamente. Cuando el síndrome se presenta tardíamente, con el paciente extubado, pueden manifestarse elementos de mala perfusión cerebral como desorientación, excitación psicomotriz y bradipsiquia.

Los exámenes paraclínicos también manifiestan elementos de hipoperfusión periférica, como ascenso del lactato, acidosis metabólica, aumento de la creatininemia, elevación de troponinas, y diversas alteraciones ecocardiográficas. La radiografía de tórax puede evidenciar alteraciones que expliquen la hipoxemia o el compromiso de la precarga (edema cardiogénico o distrés pulmonares, derrame pleural, neumotórax, derrame pericárdico con aumento de la silueta cardiopericárdica). El electrocardiograma puede presentar signos de isquemia, infarto de miocárdico y arritmias.

El cuadro clínico suele ser muy variado, y depende, en gran parte, de la etiología del mismo. Al contrario de la presentación "clásica", se constata bradicardia en vez de taquicardia cuando la etiología es bloqueo auriculoventricular completo, así como se puede evidenciar una marcada hipoperfusión con extremidades calientes, en los escenarios de síndrome vasopléjico y/o shock séptico. La presión venosa central (PVC) puede estar muy aumentada, con PCP normal cuando se produce un taponamiento cardíaco por derrame libre en cavidad pericárdica, o con PCP elevada en el contexto de infarto de mio-
Tabla 2. Etiología del síndrome de bajo gasto cardíaco.

Disminución de la precarga

- Hipovolemia

- Sangrado aumentado - discrasia (taponamiento cardíaco)

- Ventilación con presión positiva

- Dilatación ventricular derecha

- Neumotórax a tensión

Aumento de la poscarga

Aumento de las resistencias vasculares sistémicas

- Sobrecarga de volumen (aumento de tensión parietal)

- Distensión ventricular

- Obstrucción del tracto de salida del ventrículo izquierdo

- Hipertensión pulmonar

Ritmo y frecuencia cardíaca

- Taquicardia con reducción del tiempo de llenado cardíaco

- Asincronía aurículoventricular

- Bradicardia

- Arritmias auriculares con pérdida de la contracción auricular.

- Arritmias ventriculares

Disminución de la contractilidad

- Fracción de eyección del ventrículo izquierdo preoperatoria baja

- Disfunción miocárdica

- Protección miocárdica intraoperatoria inadecuada

- Isquemia - reperfusión

- Revascularización incompleta

- Complicaciones en la anastomosis

- Espasmo de la arteria coronaria nativa o del injerto.

- Infarto agudo perioperatorio

- Hipoxia, hipercapnia, acidosis

- Aumento de la poscarga

Disfunción diastólica

- Síndromes asociados con inestabilidad cardiovascular e hipotensión

- Sepsis

- Síndrome vasopléjico

- Reacción anafiláctica

- Insuficiencia suprarrenal

- Reacciones a la protamina 
cardio perioperatorio. La función renal puede estar normal, ya que el ascenso de la creatininemia muchas veces es tardío ${ }^{(8)}$.

Por ello, el manejo apropiado requiere la consideración y detección precoz de los elementos mencionados, para individualizar el tratamiento, con una actuación rápida que minimice las consecuen$\operatorname{cias}^{(8)}$.

\section{Manejo del paciente con síndrome de bajo} gasto cardíaco

El objetivo del manejo hemodinámico en el PO de cirugía cardíaca es satisfacer la demanda metabólica tisular de oxígeno, logrando un adecuado volumen minuto, y evitando que el corazón consuma niveles elevados de oxígeno. Para ello es necesario tener en cuenta los factores predisponentes, conocer los detalles del intraoperatorio, y realizar una monitorización clínica y paraclínica de los parámetros que evalúan el tratamiento instituído durante el PO.

Se deben evaluar de forma permanente los determinantes del GC: precarga, poscarga, contractilidad y frecuencia cardíaca (FC), así como la respuesta al tratamiento instaurado. Para ello, debemos tener en cuenta la clínica, así como también ciertos parámetros hemodinámicos obtenidos por medio de sistemas de monitoreo, que pueden ser invasivos o no, aunque el uso rutinario de estos no ha demostrado una reducción de la mortalidad ${ }^{(5)}$.

Si bien en la práctica habitual no se utilizan algunas técnicas de monitoreo invasivo, para entender los conceptos clave del tratamiento, es importante conocer los parámetros que de ellas se obtienen, para lograr un adecuado GC en los diferentes escenarios clínicos.

\section{Monitoreo hemodinámico}

En los últimos años, el monitoreo hemodinámico ha evolucionado a técnicas menos invasivas, pasando de ser medidas intermitentes a continuas y permitiendo cuantificar el GC y las presiones intracardíacas. Ninguna de ellas es ideal; cada una tiene ventajas y desventajas, y su uso depende de su disponibilidad, costo, y eventuales complicaciones ${ }^{(22)}$. Mencionaremos algunas de ellas.

\section{Ecocardiograma}

Es uno de los métodos más utilizados en la práctica diaria. En su versión transtorácica, es un método no invasivo que permite conocer los volúmenes cardíacos, la función de ambos ventrículos, la etiología del SBGC (disfunción sistólica, diastólica, del VD o del VI), estimar la precarga (aunque con ciertas limita- ciones) y la respuesta a la administración de volumen. El ecocardiograma transesofágico (ETE) puede ser beneficioso cuando la información no pueda obtenerse con el ecocardiograma transtorácico (ETT), como por ejemplo en caso de alta sospecha de taponamiento cardíaco ${ }^{(5,6,8)}$. Como desventajas, es un método operador dependiente y no permite obtener un monitoreo continuo.

Hay otros métodos que utilizan el utrasonido, entre los cuales se destaca el ETE para monitoreo hemodinámico, que consiste en una pequeña sonda transesofágica que permite obtener imágenes cardíacas monoplanares, o el monitoreo del GC ultrasónico (USCOM por sus siglas en inglés) que proporciona imágenes del tracto de salida aórtico y pulmonar por medio de una ventana supraesternal o intercostal.

\section{Catéter de Swan-Ganz}

Es un catéter arterial pulmonar que permite calcular el GC por técnica de termodilución, y mide la presión en la arteria pulmonar (PAP), la PCP y la PVC. Este método aún es considerado el gold standard para la medición del GC. Tiene el inconveniente de ser un método invasivo, no exento de complicaciones infecciosas y trombóticas y riesgo de lesión de la arteria pulmonar, por lo que últimamente ha sido reemplazado por métodos menos invasivos como el ecocardiograma. Su uso no ha demostrado reducir la morbilidad ni la mortalidad quirúrgica. En un estudio retrospectivo publicado en 2014, con pacientes intervenidos del 2000 al 2010, no ha demostrado ser superior en cuanto a morbilidad y mortalidad en comparación con pacientes en los que no se implantó el catéter ${ }^{(23)}$. Por otro lado, podría ser beneficioso en aquellos pacientes con shock cardiogénico no respondedores al tratamiento, o con falla del $\mathrm{VD}^{(6)}$.

Otros métodos menos invasivos que permiten el monitoreo continuo del GC mediante el análisis de la onda del pulso arterial y utilizan la termodilución para su calibración sin necesidad de implantar un catéter pulmonar son el PiCCO PulsioFlex y el VolumeView/EV1000. El uso de estos métodos está limitado por su costo ${ }^{(6)}$ y por su menor precisión en relación a los métodos por termodilución ${ }^{(6)}$.

Los sistemas FloTRac/Vigileo, ProAQT/PulsioFlex y LiDCOrapid son ejemplos de métodos mínimamente invasivos, que a través del análisis de la forma de la onda del pulso radial o femoral proporcionan un monitoreo continuo del GC sin necesidad de recurrir a la termodilución para su calibración ${ }^{(6,22)}$.

Además del ETT, dentro de los estudios no invasivos se encuentra la biorreactancia eléctrica torácica, que analiza los cambios de amplitud y frecuencia 
de los impulsos eléctricos a nivel torácico ${ }^{(22)}$. No es utilizada en la práctica clínica ya que habitualmente los pacientes tienen un catéter arterial que permite utilizar métodos mínimamente invasivos ${ }^{(6)}$.

\section{Medición de presión venosa central}

Se obtiene colocando un catéter en la aurícula derecha $(\mathrm{AD})$ o venas intratorácicas para evaluar la precarga del VD, pudiéndose correlacionar con la precarga del VI. A su vez, este catéter permite la perfusión de medicación y el análisis de la saturación venosa central de $\mathrm{O}_{2}{ }^{(5)}$.

\section{Saturación venosa de oxígeno}

Si bien no es un parámetro hemodinámico, la saturación venosa mixta de oxígeno $\left(\mathrm{SvO}_{2}\right)$ es un indicador de la perfusión de los tejidos. La verdadera $\mathrm{SvO}_{2}$ se obtiene de la arteria pulmonar, pero la muestra tomada de un catéter venoso central se acepta como válida en la práctica clínica habitual (aunque pueden diferir entre sí en pacientes sometidos a cirugía cardíaca ${ }^{(25)}$. Valores de $\mathrm{SvO}_{2}<60 \%$ o $>77 \%$, se consideran marcadores de mal pronóstico tras la cirugía de revascularización miocárdica ${ }^{(26,27)}$. La mejoría de la $\mathrm{SvO}_{2}$ luego de la administración de volumen, puede reflejar un aumento del GC, aunque la correlación entre ambos es débil ${ }^{(8,28)}$. Este parámetro no suele determinarse de forma rutinaria ${ }^{(5)}$.

\section{Lactato}

La hiperlactatemia es un marcador de hipoxia tisular. Se considera un factor pronóstico en pacientes críticos, y se ha utilizado, en combinación con la $\mathrm{SvO}_{2}$, para guiar el tratamiento en pacientes pos-cirugía cardíaca ${ }^{(5,6)}$.

\section{Optimización de los determinantes del gasto cardíaco}

El tratamiento del SBGC es muy complejo y varía en cada paciente. Depende en gran medida de la causa principal que provoca la caída del GC, pero también de otros factores tales como las condiciones cardiovasculares preoperatorias (FEVI, dilatación o no de cavidades, HTP), fenómenos acompañantes que pueden comprometer aún más la condición clínica del paciente y la respuesta al tratamiento instituido. Dada la dificultad en abarcar todas las etiologías, resulta práctico abordar el tema de forma conceptual, centrándonos en los determinantes del GC que debemos corregir.

\section{Precarga}

La optimización de la precarga de ambos ventrículos es uno de los primeros objetivos en el paciente con SBGC. Se define como la longitud de la fibra miocárdica al final de la diástole, aunque en la práctica medimos las presiones intracavitarias o el volumen de fin de diástole ${ }^{(8,29)}$.

La hipovolemia( ${ }^{(8)}$ y también la presión positiva al final de la espiración (PEEP) elevada ${ }^{(24)}$ son causas de disminución de la precarga, por lo que la administración de volumen y el descenso de la PEEP pueden mejorar el llenado ventricular.

Sin embargo, el aumento de la precarga no siempre aumenta el volumen sistólico. La respuesta a la carga de volumen es individual y depende de la curva de función ventricular (curva de Frank-Starling), la cual a su vez depende del grado de contractilidad y poscarga ventriculares ${ }^{(29)}$. Para estimar la precarga, contamos con medidas estáticas y dinámicas $^{(29)}$. La precarga del VD se estima con la medición de la $\mathrm{PVC}^{(5)}$. Se debe corregir de forma cuidadosa, debido a los efectos deletéreos de la sobredilatación de esta cavidad. La presión en la AD no debería ser $>20 \mathrm{mmHg}^{(8)}$. Por otro lado, la precarga excesiva aumenta la poscarga ventricular, sobre todo en el VD, aumentando la demanda de oxígeno, y comprometiendo tanto la perfusión coronaria como la contractilidad. A su vez, puede generar hipertensión venosa sistémica y reducir la presión de perfusión a otros órganos ${ }^{(8)}$.

Las presiones de llenado del VI se estiman con un catéter de Swan-Ganz en la arteria pulmonar, midiendo la PAP y la PCP. En la práctica habitual no se coloca el catéter y se utiliza la medición de la PVC por medio de un catéter en la $\mathrm{AD}$ o venas intratorácicas $^{(8)}$. Se sugiere que si la PVC es $>15-18$ $\mathrm{mmHg}$, se debería usar inotrópicos (aunque en corazones dilatados, la PVC es imprecisa para la estimación de la precarga del VI) ${ }^{(8)}$.

En caso de usar un catéter de Swan-Ganz, la PCP óptima debería situarse entre 15-18 mmHg, aunque en ventrículos rígidos, como sucede en la HVI, sería mejor una PCP cercana a $20 \mathrm{mmHg}$. Por el contrario, los corazones dilatados, muy distensibles, pueden presentar volúmenes de fin de diástole aumentados con presiones más bajas. Si bien la PAP, en general, se relaciona con la PCP y hace innecesario enclavar el catéter, en pacientes con HTP o enfermedad pulmonar, la primera puede ser mayor que la segunda, por lo que la PAP sobrestimaría los volúmenes del $\mathrm{VI}^{(8)}$. Debemos mencionar también que la PAP y la PCP se correlacionan poco con el volumen de fin de diástole del VI en el PO temprano, debido a alteraciones en la distensibilidad ventricular por edema ${ }^{(8)}$.

Sin embargo, estos parámetros estáticos se correlacionan poco con la capacidad de respuesta al volumen. Es importante valorar qué ocurre con el 
volumen sistólico tras la administración de volumen. Para eso se utilizan medidas dinámicas como la variación de la presión de pulso arterial inducida por la ventilación mecánica, o la respuesta a medidas posturales, como la elevación de miembros inferiores, que discriminan en forma adecuada, entre los pacientes que se beneficiarían del aporte de volumen, de aquellos en quienes sería más adecuado el uso de inotrópicos ${ }^{(29)}$. Se ha visto que un aumento en la $\mathrm{SvO}_{2} \geq 2 \%$ tras el aporte de volumen, se correlaciona con un aumento del $\mathrm{GC}^{(28)}$.

\section{Poscarga}

Conceptualmente es la resistencia a la cual los ventrículos deben enfrentarse durante la sístole. Tiene relación directa con la tensión de la pared ventricular durante la sístole. En el VD la poscarga aumentada puede deberse a aumento del volumen del líquido extravascular pulmonar, hipertensión o embolia pulmonares, ventilación por presión positiva, transfusiones de sangre y espasmo vascular pulmonar reversible ${ }^{(6,8)}$. En algunos casos la HTP puede disminuirse cambiando el inotrópico que se está utilizando por otro con mayor efecto vasodilatador pulmonar, como dobutamina o milrinona. Se debe también corregir la hipercapnia y acidosis que aumentan las resistencias vasculares pulmonares. A su vez, se debe optimizar el volumen corriente y la PEEP, ya que ambas aumentan la $\operatorname{PAP}^{(24)}$.

A nivel del VI, el aumento de la poscarga que provoca una reducción del GC, puede deberse al aumento de las RVS (aunque a veces puede ser un mecanismo compensador para mantener la presión de perfusión a los órganos). Si la RVS es $>1.500$ $\mathrm{dyn} / \mathrm{s} / \mathrm{cm}^{-5}$ se podría indicar vasodilatadores solos o asociados a inotrópicos. Otra causa de aumento de la poscarga del VI es una obstrucción en el TSVI, fija (como en la estenosis aórtica), o dinámica (como puede ocurrir en pacientes con HVI, aumento de la contractilidad y de la FC por inotrópicos, con reducción del tiempo de llenado diastólico). En caso de gradiente aumentado, una disminución de la dosis de inotrópicos, o incluso su suspensión, puede mejorar el cuadro ${ }^{(8,17)}$.

\section{Ritmo y frecuencia cardíaca}

Las alteraciones de la conducción y la FC pueden agravar la inestabilidad hemodinámica ${ }^{(5)}$. Es necesario mantener la sincronía auriculoventricular, que permite un adecuado llenado ventricular al final de la diástole, mejorando la precarga y el GC. En ocasiones es necesaria la cardioversión eléctrica o farmacológica para retomar el ritmo sinusal ${ }^{(5)}$.

La FC es un determinante directo del GC $(\mathrm{GC}=$ volumen sistólico x FC), pero su aumento excesivo puede comprometer el llenado diastólico (menor volumen diastólico final y menor volumen sistólico) y aumentar el consumo miocárdico de oxígeno (determinando disfunción miocárdica sistólica) con la consiguiente disminución del gasto. Puede ser necesario el control de frecuencia con fármacos, en el caso de taquiarritmias supraventriculares. Las bradiarritmias también pueden provocar SBGC, que se corrige por medio de la estimulación epicárdica con marcapasos transitorio. Las taquiarritmias ventriculares requieren cardioversión. La FC debe optimizarse según las características clínicas de cada paciente. Los pacientes con ventrículos pequeños con HVI u obstrucción del TSVI, se benefician de tiempos de llenado diastólico prolongados y, por ende, de FC más bajas. Los pacientes con dilatación ventricular (sobre todo derecha) que aumenta la tensión parietal (poscarga), se benefician de FC elevadas. En algunos casos de bloqueo auriculoventricular o bradicardia significativa, es necesario el uso de marcapasos o inotrópicos como el isoproterenol.

\section{Contractilidad}

Es la capacidad intrínseca del miocito de contraerse, independientemente de la precarga y la poscarga. La disfunción sistólica en el PO de cirugía cardíaca, como se ha comentado previamente, es muchas veces multifactorial. Cuando el compromiso de la función sistólica persiste (lo cual es frecuente a pesar de optimizar pre y poscarga), está indicado el uso de inotrópicos, y en ocasiones se debe utilizar un dispositivo de asistencia ${ }^{(5,8)}$. El uso de inotrópicos debe ser individualizado, ya que lo que puede beneficiar a unos, puede ser deletéreo para otros.

\section{Dispositivos de asistencia circulatoria mecánica}

En ocasiones la respuesta a la terapia con fármacos y fluidos resulta ineficaz para mantener un GC adecuado, y debemos recurrir a dispositivos de asistencia circulatoria mecánica. Brevemente, mencionaremos algunos aspectos.

\section{Balón de contrapulsación intra-aórtico}

El BIAC es uno de los dispositivos más utilizados por ser efectivo y económico. Reduce la demanda miocárdica de oxígeno al disminuir la poscarga. A su vez, mediante el aumento de la presión diastólica, aumenta la perfusión coronaria, con el aumento resultante del GC. Se recomienda en pacientes que no pueden desvincularse de la CEC, o en aquellos con SBGC refractarios al tratamiento convencional ${ }^{(5,8)}$. También está indicado en complicaciones mecáni- 
cas del infarto de miocardio como la insuficiencia mitral aguda y comunicación interventricular y en arritmias ventriculares refractarias ${ }^{(30)}$. Bajo algunas circunstancias, se coloca en casos de disfunción diastólica izquierda, mientras que su uso en pacientes con disfunción ventricular derecha es controversial, con algunos estudios que muestran beneficio $^{(30,31)}$.

\section{Dispositivos de asistencia ventricular}

Existen otros dispositivos de asistencia circulatoria mecánica que proporcionan soporte hemodinámico ventricular izquierdo, derecho o biventricular. Algunos ejemplos son el dispositivo de tipo oxigenador de membrana (ECMO por sus siglas en inglés), y el sistema TandemHeart ${ }^{\circledR}$ o el Impella ${ }^{\circledR}$. Se recomienda en los pacientes que no pueden desvincularse de la CEC o que desarrollan shock cardiogénico en el PO inmediato, pese al tratamiento convencional y/o BIAC(5)

El objetivo es mejorar el estado del paciente para eventualmente desvincularlo del dispositivo ("puente a la recuperación") o servir de soporte hemodinámico como "puente al trasplante" cardíaco, para lo cual es fundamental valorar la función del VD(5).

\section{Conclusiones}

La presencia de SBGC en el PO de cirugía cardíaca se asocia a morbimortalidad aumentada y prolonga la estadía en terapia intensiva. Es fundamental conocer la fisiopatología del cuadro y tener en mente las posibles causas que lo provocan, así como sus consecuencias para un manejo óptimo. Se debe reconocer precozmente, individualizar el tratamiento y evaluar de forma constante la respuesta a este, para minimizar sus consecuencias y mejorar la evolución posoperatoria de nuestros pacientes.

\section{Sebastián Lorenzo, \\ https://orcid.org/0000-0003-2827-601X}

Este artículo fue aceptado para su publicación por: Editor asociado Dr. Jorge Estigarribia

\section{Bibliografia}

1. Chandler H, Kirsch R. Management of the low cardiac output syndrome following surgery for congenital heart disease. Curr Cardiol Rev. 2016; 12(2):107-11. doi: 10.2174/1573403x1266615111916 4647

2. Rao V, Ivanov J, Weisel R, Ikonomidis J, Christakis G, David T. Predictors of low cardiac output syndrome after coronary artery bypass. J Thorac Cardiovasc Surg. 1996; 112(1):38-51. doi: 10.1016/ s0022-5223(96)70176-9

3. Fernández J, Padrón K, Paredes Á, Vázquez E, González O, González A. Predictores de bajo gasto cardíaco perioperatorio en pacientes operados de cirugía cardíaca valvular. CorSalud. 2018; 10(4):28693.

4. Ciruzzi M, Henquin R, Aranda G, Bozovich G, Heredia P, Rodríguez $\mathbf{R}$, et al. CONAREC III: evolución de los pacientes sometidos a cirugía coronaria . Estudio multicéntrico. Rev Argent Cardiol. 1996; 64(1): 91-100.

5. Pérez Vela J, Martín Benítez J, Carrasco González M, de la Cal López M, Hinojosa Pérez R, Sagredo Meneses V, et al. Guías de práctica clínica para el manejo del síndrome de bajo gasto cardíaco en el postoperatorio de cirugía cardíaca. Med Intensiva. 2012; 36(4):e1-e44. doi: 10.1016/j.medin.2012.02.007

6. Lomivorotov V, Efremov S, Kirov M, Fominskiy E, Karaskov A. Low-cardiac-output syndrome after cardiac surgery. J Cardiothorac Vasc Anesth. 2017; 31(1):291-308. doi: 10.1053/j.jvca.2016.05.029

7. Noel-Morgan J, Muir W. Anesthesia-associated relative hypovolemia: mechanisms, monitoring, and treatment considerations. Front Vet Sci. 2018; 5:53. doi: $10.3389 /$ fvets. 2018.00053

6. Bojar RM. Early postoperative care. En: Management manual of perioperative care in adult cardiac surgery. 5th ed. Massachusetts: Blackwell; 2005. Cap.11

9. Ding W, Ji Q, Shi Y, Ma R. Predictors of low cardiac output syndrome after isolated coronary artery bypass grafting. Int Heart J. 2015; 56(2):144-9. doi: 10.1536/ihj.14-231

10. Algarni K, Maganti M, Yau T. Predictors of low cardiac output syndrome after isolated coronary artery bypass surgery: trends over 20 years. Ann Thorac Surg. 2011; 92(5):1678-84. doi: 10.1016/j.athoracsur.2011.06.017

11. Lomivorotov V, Efremov S, Boboshko V, Nikolaev D, Vedernikov P, Lomivorotov V, et al. Evaluation of nutritional screening tools for patients scheduled for cardiac surgery. Nutrition. 2013; 29(2):436-42. doi: 10.1016/j.nut.2012.08.006

12. Tolpin D, Collard C, Lee VV, Elayda M, Pan W. Obesity is associated with increased morbidity after coronary artery bypass graft surgery in patients with renal insufficiency. J Thorac Cardiovasc Surg. 2009; 138(4):873-9. doi: 10.1016/j.jtcvs.2009.02.019

13. Hernández-Leiva E, Dennis R, Isaza D, Umaña J. Hemoglobin and B-type natriuretic peptide preoperative values but not inflammatory markers, are associated with postoperative morbidity in cardiac surgery: a prospective cohort analytic study. J Car- 
diothorac Surg. 2013; 8:170. doi: 10.1186/1749- 80908-170

14. Lomivorotov V, Efremov S, Boboshko V, Leyderman I, Lomivorotov VN, Cheung A, et al. Preoperative total lymphocyte count in peripheral blood as a predictor of poor outcome in adult cardiac surgery. J Cardiothorac Vasc Anesth. 2011; 25(6):975-80. doi: 10.1053/j.jvca.2010.12.006

15. Paparella D, Cappabianca G, Visicchio G, Galeone A, Marzovillo A, Gallo N, et al. Cardiac troponin I release after coronary artery bypass grafting operation: effects on operative and midterm survival. Ann Thorac Surg. 2005; 80(5):1758-64. doi: 10.1016 j.athoracsur.2005.04.040

16. Epting C, McBride M, Wald E, Costello J. Pathophysiology of Post-Operative Low Cardiac Output Syndrome. Curr Vasc Pharmacol. 2016; 14(1):14-23. doi: 10.2174/1570161113666151014123718

17. Reyes-Sánchez M, Carrillo-Rojas J, Hernández-Mercado M, Amaro-Camacho J, Herrera-Garza E, López-Pineda D, et al. Síndrome de bajo gasto cardíaco postcardiotomía. Arch Cardiol Mex. 2011; 81(Supl 2):30-40.

18. Sánchez-Hernández C, Torres-Alarcón L, González-Cortés A, Peón A. Ischemia/reperfusion injury: pathophysiology, current clinical management, and potential preventive approaches. Mediators Inflamm. 2020; 2020:8405370. doi: 10.1155/2020/ 8405370

19. Krauss J. Preoperatorio y posoperatorio en cirugía cardíaca. En: Bertolasi C, Barrero C, Gimeno G, Liniado G, Mauro V. Bertolasi: Cardiología 2000. 2 ed. Buenos Aires: Panamericana; 1998 p. 1443-70.

20. Bernard F, Denault A, Babin D, Goyer C, Couture P, Couturier A, et al. Diastolic dysfunction is predictive of difficult weaning from cardiopulmonary bypass. Anesth Analg. 2001; 92(2):291-8. doi: 10. 1097/00000539-200102000-00002

21. Casthely P, Shah C, Mekhjian H, Swistel D, Yoganathan T, Komer C, et al. Left ventricular diastolic function after coronary artery bypass grafting: a correlative study with three different myocardial protection techniques. J Thorac Cardiovasc Surg. 1997; 114(2):254-60. doi: 10.1016/S0022-5223(97) 70153-3

22. Mateu Campos M, Ferrándiz Sellés A, Gruartmoner de Vera G, Mesquida Febrer J, Sabatier Cloarec C, Poveda Hernández Y, et al. Técnicas disponibles de monitorización hemodinámica: venta- jas y limitaciones. Med Intensiva. 2012; 36(6):434-44. doi: 10.1016/j.medin.2012.05.003

23. Chiang Y, Hosseinian L, Rhee A, Itagaki S, Cavallaro P, Chikwe J. Questionable benefit of the pulmonary artery catheter after cardiac surgery in high-risk patients. J Cardiothorac Vasc Anesth. 2015; 29(1):76-81. doi: 10.1053/j.jvca.2014.07.017

24. Jardin F, Vieillard-Baron A. Right ventricular function and positive pressure ventilation in clinical practice: from hemodynamic subsets to respirator settings. Intensive Care Med. 2003; 29(9):1426-34. doi: 10.1007/s00134-003-1873-1

25. Lorentzen A, Lindskov C, Sloth E, Jakobsen C. Central venous oxygen saturation cannot replace mixed venous saturation in patients undergoing cardiac surgery. J Cardiothorac Vasc Anesth. 2008; 22(6):853-7. doi: 10.1053/j.jvca.2008.04.004

26. Holm J, Håkanson E, Vánky F, Svedjeholm R. Mixed venous oxygen saturation predicts short- and long-term outcome after coronary artery bypass grafting surgery: a retrospective cohort analysis. $\mathrm{Br} \mathrm{J}$ Anaesth. 2011; 107(3):344-50. doi: 10.1093/ bja/aer 166

27. Perz S, Uhlig T, Kohl M, Bredle D, Reinhart K, Bauer M, et al. Low and "supranormal" central venous oxygen saturation and markers of tissue hypoxia in cardiac surgery patients: a prospective observational study. Intensive Care Med. 2011; 37(1):52-9. doi: 10.1007/s00134-010-1980-8

28. Kuiper A, Trof R, Groeneveld A. Mixed venous O2 saturation and fluid responsiveness after cardiac or major vascular surgery. J Cardiothorac Surg. 2013; 8:189. doi: 10.1186/1749-8090-8-189

29. Sabatier C, Monge I, Maynar J, Ochagavía A. Valoración de la precarga y la respuesta cardiovascular al aporte de volumen. Med Intensiva. 2012; 36(1):45-55

30. Boeken U, Feindt P, Litmathe J, Kurt M, Gams E. Intraaortic balloon pumping in patients with right ventricular insufficiency after cardiac surgery: parameters to predict failure of IABP Support. Thorac Cardiovasc Surg. 2009; 57(6):324-8. doi: 10.1055/s0029-1185766

31. Liakopoulos O, Ho J, Yezbick A, Sanchez E, Singh V, Mahajan A. Right ventricular failure resulting from pressure overload: role of intra-aortic balloon counterpulsation and vasopressor therapy. $J$ Surg Res. 2010; 164(1):58-66. doi: 10.1016/j.jss. 2009.04.044 